\title{
Comparaison des cinétiques de coagulation enzymatique et mixte du lait. Influence du calcium
}

\author{
Y. Noël ${ }^{1}$
}

\author{
avec la collaboration technique de N. Lehembre 2 , A. Dulac ${ }^{2}$ et M.C. Clavaud 2 \\ 1 INRA, station de recherches en technologie et analyses laitières, BP 89, 39800 Poligny; \\ 2 INRA, laboratoire de génie des procédés alimentaires, 25 avenue de la République, 91300 Massy, \\ France
}

(reçu le 10 novembre 1988, accepté le 27 juin 1989)

\begin{abstract}
Résumé - Les coagulations enzymatique et mixte du lait sont étudiées avec une méthode viscoélasticimétrique. Alors que les cinétiques de coagulation enzymatique comportent 2 étapes, les cinétiques de coagulation mixte en font apparaître $4: 1$ ) une phase de latence qui correspond à l'hydrolyse enzymatique et à l'agrégation des micelles, suivie par 2) la formation et le raffermissement du coagulum, puis 3) une phase de diminution de la fermeté associée à la déminéralisation du réseau protéique, enfin 4) une nouvelle phase d'augmentation de la fermeté, associée à une réorganisation de la structure du coagulum. L'influence du calcium est examinée par comparaison entre les 2 types de coagulation, mixte et enzymatique, sur les différents paramètres descriptifs : temps de latence, fermeté, vitesse de coagulation et indice de viscoélasticité.
\end{abstract}

coagulation enzymatique - coagulation mixte - propriétés viscoélasticimétriques - calcium

Summary - Comparison between enzymatic and mixed enzymatic and lactic acid coagulation of milk. Influence of calcium addition. Milk enzymatic coagulation and its combination with lactic acidification were studied using a viscoelasticimetric method. While two phases characterize enzymatic coagulation, four steps were distinguished for the combined enzymatic and lactic coagulation : 1. a lag phase during which proteolysis and micelle aggregation occurred, 2. gel formation and firming, 3. a decrease in firmness due to the demineralization of the protein structure, 4. a second increase in firmness associated with a reorganization of the structure of the coagulum. Calcium influence was examined by comparing the two coagulation types, using descriptive parameters : lag phase, firmness, coagulation rate, viscoelasticity index.

enzymatic coagulation - combined enzymatic and lactic coagulation - viscoélasticimetric properties - calcium 


\section{INTRODUCTION}

La coagulation enzymatique du lait a fait l'objet de nombreuses recherches sur lesquelles il existe plusieurs revues bibliographiques : en particulier, Thomasow \& Voss (1977), Prokopek (1978) sur les méthodes d'étude, Green (1977) sur les agents coagulants, Walstra \& Van Vliet (1986) sur la physicochimie de la coagulation. En revanche, la coagulation mixte résultant de l'action conjointe d'enzymes coagulantes et de l'acidification par les bactéries lactiques a été moins étudiée (Roefs, 1986; Van Hooydonk et al., 1986b). Trois types de difficultés peuvent expliquer cette situation : d'une part, la maîtrise des développements bactériens n'est pas acquise, d'autre part la coagulation mixte met en jeu des phénomènes physicochimiques plus complexes que la coagulation enzymatique, enfin les instruments de mesure appropriés sont rares. Or, la technologie fromagère est souvent basée sur des coagulations mixtes. II importe donc de progresser dans la connaissance des phénomènes qui leurs sont associés, connaissance particulièrement nécessaire à l'optimisation de capteurs pour le contrôle automatique en fabrication fromagère.

Nous présentons ici les premiers résultats obtenus en coagulation mixte avec le viscoélasticimètre GVT-INRA (Gervais et al., 1982a), et nous les comparons avec ceux obtenus en coagulation enzymatique. De plus, les effets de l'addition du calcium, dont le mécanisme d'action au cours des différentes étapes de la coagulation n'est pas encore complètement élucidé (Van Hooydonk et al., 1986c; Bringe \& Kinsella, 1986) ont été examinés et sur les 2 types de coagulation est discutée leur comparaison.

\section{MATÉRIELS ET MÉTHODES}

\section{Mesures rhéologiques}

Le viscoélasticimètre GVT-INRA, utilisé pour suivre les cinétiques de coagulation, fonctionne sur le principe du cisaillement harmonique en entrefer large. Les essais ont été conduits avec une amplitude du mouvement du cylindre de mesure de 4 degrés et une fréquence de $10^{-1}$ $\mathrm{Hz}$. Le mouvement du cylindre de mesure est connu, à chaque instant, grâce à un capteur potentiométrique de déplacement angulaire Bonohm (Le Prototype Mécanique Industrie, F78260 L'Etang-La-Ville). La résistance du coagulum en formation est mesurée par un capteur de couple à jauges de contrainte (Société Européenne d'Extensométrie Appliquée, F-28000 Chartres). La saisie des données est assurée par un calculateur Hewlett Packard HP9816 et un oscilloscope numérique à mémoire Nicolet Explorer 2090 IIIA (tiroir 206), accompagnés des périphériques suivants : un disque dur HP9134, une imprimante HP 2225AB et une table traçante Tektronix 4662.

Le comportement rhéologique du coagulum de lait est observé, avec le viscoélasticimètre GVT-INRA, dans le domaine non linéaire (Gervais et al., 1982b), ce qui se traduit par l'existence d'harmoniques dans le signal de réponse. L'analyse de Fourier permet alors de séparer la partie linéaire (signal fondamental) de la partie non linéaire (signaux harmoniques). On ne s'intéressera par la suite qu'à la partie linéaire du signal (Kobayashi et al., 1982).

La contrainte est calculée à partir de la mesure du moment de couple, en tenant compte des caractéristiques géométriques du cylindre et après correction de l'inertie de l'équipage mobile constitué par le cylindre et le couplemètre (Lefèvre, 1988).

On calcule enfin le déphasage entre le signal sinusoïdal du mouvement du cylindre et le signal fondamental du couple mesuré, ou angle de perte.

\section{Le lait}

Le lait utilisé pour les essais ( $5 \mathrm{~kg}$ par essai) est reconstitué dans de l'eau bidistillée à $45^{\circ} \mathrm{C}$. sous agitation, à partir d'une poudre de lait écré- 
mé Nilac (Nizo, Hollande) du type basse température, à raison de $100 \mathrm{~g}$ de poudre par $\mathrm{kg}$ de lait reconstitué. Le mélange est agité sans chauffage (1 $250 \mathrm{t} / \mathrm{min})$ pendant $1 \mathrm{~h}$.

La teneur moyenne en calcium total du lait reconstitué est de $1,30 \mathrm{~g} / \mathrm{kg}$. Les solutions de chlorure de calcium ajoutées au lait ont été préparées à partir de $\mathrm{CaCl}_{2}, 2 \mathrm{H}_{2} \mathrm{O}$ cristallisé (Prolabo).

\section{Coagulation enzymatique}

Le lait destiné aux essais en coagulation enzymatique est additionné d'un antibactérien $(1 \mathrm{ml}$ d'une solution de thiomersal à $100 \mathrm{~g} / \mathrm{l}$ pour 1 । de lait). Après reconstitution, le lait est versé dans la cuve du viscoélasticimètre GVT-INRA, où il s'équilibre au repos pendant $16-18 \mathrm{~h}$ à $30^{\circ} \mathrm{C}$ (Famelart, 1982; Roefs, 1986).

La solution enzymatique est préparée à partir d'une présure en poudre Hansen solubilisée dans un tampon acétate de sodium $0,05 \mathrm{~mol} / \mathrm{l}$ à $\mathrm{pH} 5,5$. La quantité ajoutée, soit $0,5 \mathrm{ml}$, représente $222 \mu \mathrm{g}$ de chymosine active par $\mathrm{kg}$ de lait reconstitué. L'activité chymosine de la présure utilisée représentait $99,3 \%$ de l'activité coagulante totale, déterminée selon la norme FIL 110 A (FIL, 1987).

Les apports en chlorure de calcium correspondent à 9 niveaux compris entre 0 et $400 \mathrm{mg} /$ $\mathrm{kg}$ de lait. Lors de la reconstitution, le $\mathrm{pH}$ final du lait est ajusté à 6,60 par addition d'une quantité appropriée d'une solution normale d'acide chlorhydrique ou de soude.

\section{Coagulation mixte}

Le lait destiné aux essais en coagulation mixte est reconstitué sans antibactérien, puis versé dans la cuve du viscoélasticimètre thermostatée à $30^{\circ} \mathrm{C}$; il est maintenu pendant $1 \mathrm{~h}$ avant l'addition des ferments lactiques.

Les $5 \mathrm{~kg}$ de lait sont ensemencés avec $50 \mathrm{ml}$ de ferments préparés à partir d'une souche de Streptococcus lactis ssp diacetylactis (souche INRA $n^{\circ} 125$ ). Le $\mathrm{pH}$ du lait ensemencé est suivi en continu au moyen d'un pHmètre Orion SA 720 équipé d'une électrode de $\mathrm{pH}$ Ross 81.62 $(E T=0,02)$. Lorsque le $\mathrm{pH}$ est à 6,00 (temps $t_{0}{ }_{0}$ ), la solution enzymatique, préparée comme précédemment à partir de présure en poudre Hansen, est ajoutée au lait à la dose de $88,8 \mu \mathrm{g}$ de chymosine active par $\mathrm{kg}$ de lait.

Les apports de chlorure de calcium correspondent à 6 niveaux entre 0 et $400 \mathrm{mg} / \mathrm{kg}$ de lait.

\section{RÉSULTATS}

\section{Coagulation enzymatique}

Une cinétique de coagulation enzymatique est représentée Figure 1. La courbe (1) correspond à l'évolution de la contrainte en

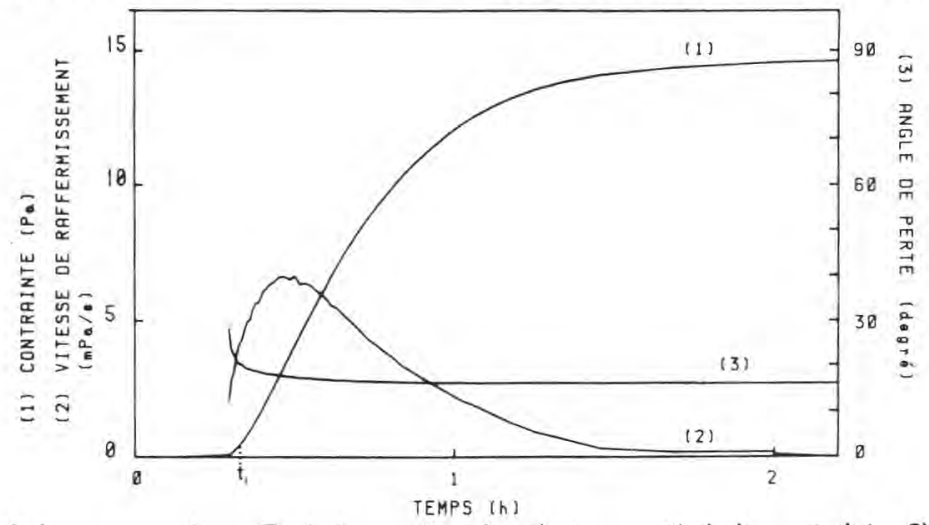

Fig. 1. Coagulation enzymatique. Evolution en fonction du temps. 1) de la contrainte, 2) de la vitesse de raffermissement, 3) de l'angle de perte. 
fonction du temps. Le temps de latence, $t_{1}$, souvent appelé "temps de gel», dont la détermination dépend du seuil de mobilité de l'appareil, est associé arbitrairement à une contrainte de $0,5 \mathrm{~Pa}$. La contrainte maximum relevée sur la cinétique correspond au plateau. La dérivée de la contrainte en fonction du temps, qui n'est autre qu'une vitesse, est aussi représentée Figure 1 (courbe 2) : le maximum de la dérivée est pris comme vitesse maximale de raffermissement. Enfin, l'évolution de l'angle de perte (courbe 3 ) est indiquée sur la même figure : on observe une diminution rapide et importante de l'angle de perte dès le début de la formation du gel, puis celui-ci tend vers une valeur limite, de l'ordre de 15 degrés d'angle.

\section{Coagulation mixte}

La Figure 2 donne un exemple de cinétique de coagulation mixte. Le temps 0 sur la figure correspond à l'addition des ferments et le temps t'o à l'addition de l'en- zyme coagulante. Après une phase de latence, on observe une augmentation de la contrainte (courbe $\mathrm{Y} 1$ ) jusqu'à un maximum $\tau_{M}$ au point $P_{M}$, puis une diminution de cette contrainte de l'ordre de $60 \%$ jusqu'à un minimum $\tau_{m}$ au point $P_{m}$ et enfin à nouveau une augmentation de la contrainte qui montre alors une tendance à se stabiliser à une valeur plateau.

La courbe Y4 indique l'évolution du pH observée dans le même temps; on remarque qu'entre le point maximum $P_{M}$ et le point minimum $P_{m}$ de la courbe de contrainte, le pH diminue de 5,60 à 5,00 .

La courbe de l'angle de perte (courbe Y3) montre une diminution très rapide jusqu'à une valeur de 15 degrés d'angle, dès le début de la formation du gel. Après un plateau assez court, l'angle de perte augmente jusqu'à un maximum qui coïncide exactement avec le minimum de la courbe de contrainte. Ensuite, l'angle de perte diminue jusqu'à une valeur seuil de l'ordre de celle qui précède le maximum.

Enfin, la courbe Y2 représente la dérivée de la contrainte en fonction du temps :

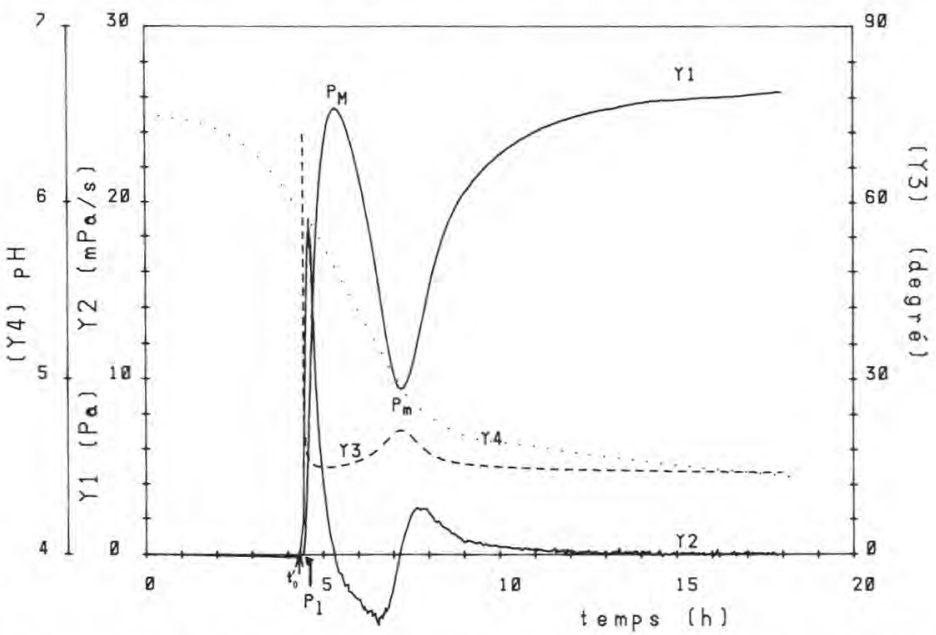

Fig. 2. Coagulation mixte. Evolution en fonction du temps. $Y_{1}$ ) de la contrainte, $Y_{2}$ ) de la vitesse de raffermissement, $\mathrm{Y}_{3}$ ) de l'angle de perte, $\left.\mathrm{Y}_{4}\right) \mathrm{du} \mathrm{pH}$. 

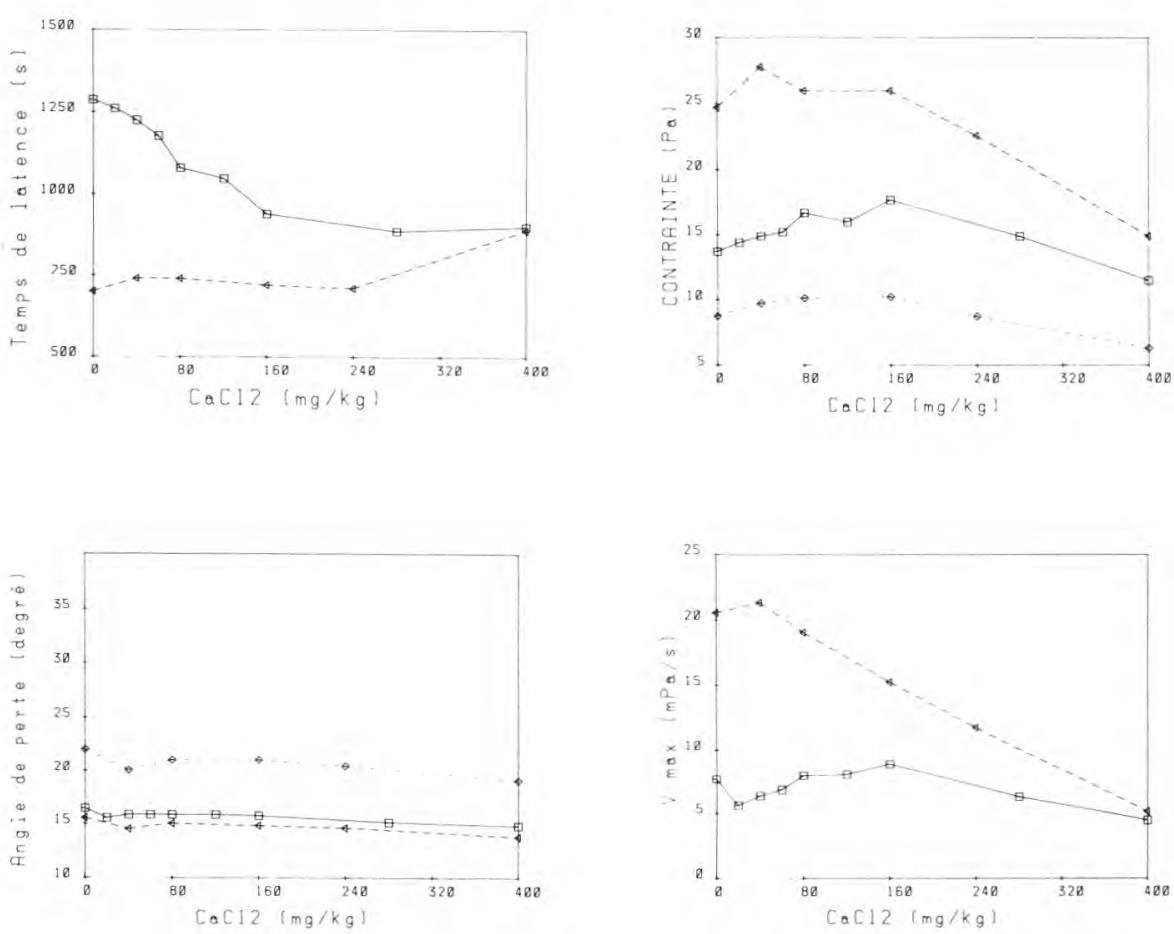

Fig. 3. Influence de l'addition du calcium sur les paramètres caractéristiques des cinétiques de coagulation enzymatique et mixte. a. Temps de latence : $\rightarrow-$ en coagulation enzymatique, - en coagulation mixte. b. Contrainte :- - -contrainte maximale en coagulation enzymatique, au point $P_{M}(--)$ et au point $\mathrm{Pm}(\cdot)$ en coagulation mixte. c. Angle de perte : $\rightarrow$ en coagulation enzymatique, au point $P_{M}\left(\cdot-*_{-}\right)$et au point $\mathrm{Pm}(-)$ en coagulation mixte. d. Vitesse maximale de raffermissement : $\rightarrow$ en coagulation enzymatique, + - en coagulation mixte.

comme pour la coagulation enzymatique, on considère le maximum de la dérivée comme la vitesse maximum de raffermissement.

\section{Addition de calcium}

L'influence de l'addition du calcium est examinée sur les principaux paramètres descriptifs de la cinétique de coagulation enzymatique ou mixte (temps de latence, contrainte maximale, vitesse maximale de raffermissement, angle de perte). Les résultats sont présentés sur la Figure 3.

\section{Coagulation enzymatique}

Le temps de latence $t_{1}$ (Fig. 3a) diminue en fonction de la concentration de calcium jusqu'à $160 \mathrm{mg} / \mathrm{kg}$, et se stabilise ensuite au voisinage d'une valeur limite. La contrainte maximale (Fig. 3b) augmente sensiblement jusqu'à 160 mg/kg, puis diminue ensuite. La valeur limite de l'angle de perte (Fig. 3c) apparaît peu sensible à l'addition de calcium. La vitesse maximale de raffermissement diminue sensiblement entre 0 et 20 mg/kg, augmente jusqu'à 160 $\mathrm{mg} / \mathrm{kg}$, puis diminue nettement au-delà (Fig. 3d). 
La reproductibilité des mesures avait été par ailleurs évaluée sur 3 laits, additionnés de $160 \mathrm{mg}$ de calcium par $\mathrm{kg}$ de lait : les coefficients de variation observés étaient respectivement de $1 \%$ sur le temps de latence et la contrainte maximale, de $2 \%$ sur la valeur limite de l'angle de perte et de $3 \%$ sur la vitesse maximale de raffermissement.

\section{Coagulation mixte}

Dans le cas de la coagulation mixte, l'influence de l'addition du calcium est examinée sur les caractéristiques des points remarquables de la cinétique (Fig. 2, courbe Y1), c'est-à-dire :

- le point $P_{1}$ qui a pour abscisse le temps de latence $t_{1}$;

- le point maximum $\mathrm{P}_{\mathrm{M}}$;

- le point minimum $P_{m}$ de la cinétique.

Chaque point est caractérisé, d'une part par ses coordonnées (contrainte et temps) sur la cinétique de coagulation, d'autre part, par le pH et l'angle de perte correspondant. Les valeurs des temps pris en compte pour l'analyse des résultats sont données en considérant comme ori-

Tableau I. Temps $t_{M}$ et $t_{m}$ associés respectivement aux points $P_{M}$ et $P_{m}$ de la cinétique de coagulation mixte en fonction de la quantité de $\mathrm{CaCl}_{2}$ ajouté.

\begin{tabular}{rcr}
\hline $\begin{array}{c}\mathrm{CaCl}_{2} \\
(\mathrm{~g} / \mathrm{kg})\end{array}$ & $\begin{array}{c}t_{M} \\
(s)\end{array}$ & $\begin{array}{c}t_{m} \\
(s)\end{array}$ \\
\hline 0 & 3460 & 9650 \\
40 & 3900 & 10850 \\
80 & 3880 & 10540 \\
120 & 4650 & 11250 \\
240 & 5200 & 12250 \\
400 & 7200 & 14800 \\
\hline
\end{tabular}

gine le temps de l'addition de la solution enzymatique.

Le temps de latence $\left(t_{1}\right)$ n'est pas sensible à l'addition du calcium, sauf à 400 $\mathrm{mg} / \mathrm{kg}$ où l'on note une augmentation très nette (Fig. 3a). Au contraire, les temps associés aux points $P_{M}$ et $P_{m}$ augmentent avec la quantité de calcium ajouté, avec un plateau entre 40 et $80 \mathrm{mg} / \mathrm{kg}$ de $\mathrm{Ca}$ (Tableau I).

La Figure $3 \mathrm{~b}$ présente les valeurs de la contrainte aux points $P_{M}$ et $P_{m}$ en fonction de la quantité de calcium ajouté. La contrainte en $P_{M}$ augmente légèrement jusqu'à $40 \mathrm{mg} / \mathrm{kg}$ de $\mathrm{CaCl}_{2}$ puis diminue faiblement jusqu'à $160 \mathrm{mg} / \mathrm{kg}$ et très nettement ensuite. L'évolution de la contrainte en $P_{m}$ n'est pas si différente de celle en $\mathrm{P}_{\mathrm{M}}$, sauf pour $80 \mathrm{mg} / \mathrm{kg}$ de $\mathrm{CaCl}_{2}$.

L'angle de perte en $P_{M}$ et $P_{m}$ manifeste une légère tendance à la baisse lorsque la quantité de calcium ajouté augmente (Fig. $3 c$ ), avec un minimum plus prononcé vers $40 \mathrm{mg} / \mathrm{kg}$. La vitesse maximum de raffermissement, calculée sur la partie de la courbe de contrainte comprise entre $P_{1}$ et $P_{M}$, augmente un peu entre 0 et $40 \mathrm{mg} / \mathrm{kg}$ de calcium ajouté, mais diminue ensuite nettement (Fig. 3d).

La reproductibilité a été estimée dans le cadre d'un plan d'expérience dont les résultats seront présentés et discutés dans un prochain article. Les coefficients de variation des paramètres étudiés ici étaient $<5 \%$, sauf celui de la contrainte au point $P_{m}$ pour lequel il atteignait $16 \%$.

\section{DISCUSSION}

\section{Cinétiques de coagulation}

La cinétique de coagulation enzymatique, représentée par l'évolution de la contrainte 
mesurée en fonction du temps, est classique. Pendant la phase de latence où la contrainte mesurée n'est pas significativement différente de zéro, se déroulent l'hydrolyse enzymatique de la caséine kappa et l'agrégation des micelles de caséines. Puis, la formation du coagulum se traduit par l'apparition d'une contrainte significativement différente de zéro, à laquelle est associé le temps de latence ou temps de gel.

L'organisation du réseau et le raffermissement du gel se traduisent ensuite par une augmentation de la fermeté, d'abord assez rapide, puis plus lente. La contrainte tend alors vers un plateau qui matérialise un comportement rhéologique stable du coagulum puisque l'angle de perte aussi est constant. L'évolution de l'angle de perte traduit ici l'apparition et le développement d'une composante élastique importante dès le début de la formation du gel. L'existence d'un plateau atteint très rapidement suggère que l'acquisition du comportement rhéologique caractéristique du coagulum se produit très tôt après la transition liquide-gel. De plus, si la part de la composante élastique par rapport à la composante visqueuse est constante dès que l'angle de perte atteint ce plateau, l'augmentation de la contrainte, c'est-à-dire de la fermeté, observée dans le même temps, indique que les amplitudes de ces $2 \mathrm{com}$ posantes augmentent mais dans les mêmes proportions. Des résultats comparables, obtenus dans le domaine linéaire, sont présentés par Walstra \& Van Vliet (1986), ainsi que par Dejmek (1987), ce qui pourrait suggérer que l'évolution observée de l'angle de perte n'est pas affectée par le caractère linéaire ou non de l'essai harmonique. Toutefois, Lee (1986) observe, dans le domaine linéaire, une diminution régulière de l'angle de perte depuis le point de gel jusqu'à l'état d'équilibre, atteint après des temps particulièrement longs. Ces différents auteurs ne fournis- sant pas de données quantitatives sur la précision des mesures, la discussion de ces résultats contradictoires est limitée. Néanmoins l'hypothèse selon laquelle la nature des liaisons déterminant le comportement rhéologique du coagulum enzymatique n'évoluerait pas (Dejmek, 1987; Walstra \& Van Vliet, 1986) doit être considérée avec prudence.

Le recours à un essai en cisaillement harmonique dans des conditions d'amplitude, de fréquence et de température non destructives pour le gel, a permis aussi de suivre des cinétiques de coagulation mixte pendant plusieurs heures sans que l'on observe de fissures ou de synérèse. Quatre étapes à partir de l'addition de l'enzyme coagulante ont ainsi été mises en évidence. Van Hooydonk et al. (1986b) ont présenté un résultat qui suggère également l'existence de plusieurs étapes, mais celles-ci n'apparaissaient pas nettement différenciées.

Les 2 premières parties de la cinétique de coagulation mixte correspondent aux 2 premières étapes de l'action de l'enzyme coagulante : hydrolyse enzymatique de la caséine kappa et agrégation des micelles pendant le temps de latence, puis coagulation proprement dite avec formation du réseau protéique et augmentation de la fermeté de celui-ci. La diminution de la contrainte que l'on observe ensuite, qui traduit une diminution de fermeté, peut être imputée à la déminéralisation du réseau protéique liée à l'évolution du $\mathrm{pH}$. En effet, on sait qu'à $\mathrm{pH}=5,10$, la déminéralisation des caséines est presque complète (Evenhuis \& De Vries, 1959; Heertje et al., 1985; Van Hooydonk et al., 1986a).

Après le point minimum $\mathrm{P}_{\mathrm{m}}$, la remontée de la contrainte et par suite de la fermeté pourrait être expliquée par la mise en place de liaisons nouvelles du type de celles d'un coagulum lactique.

L'évolution de l'angle de perte pendant les 2 premières parties est similaire à celle 
que l'on observe en coagulation enzymatique. En revanche, au voisinage du point $P_{m}$, la variation de l'angle de perte signifie que la composante visqueuse a sensiblement augmenté au détriment de la composante élastique, révélant ainsi une réorganisation de la structure du réseau. La valeur de l'angle de perte atteinte au niveau des zones "plateau" (14-15 degrés) est légèrement inférieure à celle qui est observée pour le coagulum obtenu par voie enzymatique (15-16 degrés), mais elle est nettement différente de la valeur de 25 degrés mesurée pour un coagulum lactique (Lehembre, 1986). Walstra \& Van Vliet (1986) montrent une évolution similaire de l'angle de perte en fonction du $\mathrm{pH}$. $\mathrm{Si}$ l'on retient l'hypothèse émise par certains auteurs (Walstra \& Van Vliet, 1986; Roefs, 1986; Bohlin et al., 1984) selon laquelle l'angle de perte rendrait compte du type de liaisons spécifiques d'un coagulum, nos résultats suggèreraient que la structure d'un coagulum mixte est assez proche de celle d'un coagulum enzymatique. Cependant, il convient aussi d'envisager l'hypothèse selon laquelle ce paramètre ne discriminerait pas suffisamment les différences de structure.

\section{Addition de calcium}

La discussion des résultats relatifs à l'influence comparée du calcium en coagulation enzymatique et en coagulation mixte doit être précédée de 2 remarques. Les 2 expérimentations avaient été réalisées dans des conditions différentes, notamment avec des concentrations en présure et un mode de préparation du lait différents. Toutefois, l'intérêt de faire une analyse comparée des résultats a conduit à rapprocher les 2 séries d'expérimentation. On peut raisonnablement supposer que l'incidence de la différence de concentra- tion en agent coagulant porte surtout sur les valeurs absolues des paramètres de temps, mais que les hypothèses formulées à partir des observations relatives à l'action du calcium restent valables. La seconde remarque porte sur les différences de préparation des échantillons. La coagulation par voie enzymatique autorise l'utilisation d'un antibactérien et par suite un temps d'équilibrage long à la température de l'essai (16 h). En coagulation mixte, cette procédure étant exclue, le temps d'équilibrage a été limité à $1 \mathrm{~h}$ à la température de l'essai avant d'ajouter les ferments lactiques. Des mesures de $\mathrm{pH}$ effectuées par ailleurs en laboratoire montrant que le $\mathrm{pH}$ à $1 \mathrm{~h}$ est peu différent de celui mesuré à $24 \mathrm{~h}$ : en conséquence, on supposera que les équilibres minéraux, notamment calcique, sont pratiquement réalisés. Enfin, le $\mathrm{pH}$ du lait a été corrigé après l'addition du $\mathrm{Ca}$ dans le cas de la coagulation enzymatique; cette correction n'a pas été faite en coagulation mixte mais l'emprésurage a toujours été effectué au même pH de 6,00.

On sait que l'addition de calcium à $\mathrm{pH}$ constant n'a pas d'influence sur la réaction d'hydrolyse enzymatique, mais qu'elle accélère la réaction d'agrégation des micelles hydrolysées (Van Hooydonk et al., $1986 c$ ), probablement en provoquant spécifiquement une diminution du potentiel $\zeta$ en valeur absolue (Dalgleish, 1984), ce qui se traduit par une diminution du temps de latence (Van Hooydonk et al., 1986c). Toutefois, cet effet n'est observable que pour les quantités limitées de $\mathrm{CaCl}_{2}$ ajouté : au-delà de $200 \mathrm{mg} / \mathrm{kg}$, l'addition de calcium ne modifie plus le temps de latence (Van Hooydonk, 1987).

En coagulation enzymatique, le temps de latence diminue avec la quantité de calcium ajouté jusqu'à $160 \mathrm{mg} / \mathrm{kg}$ : la diminution est lente entre 0 et $40 \mathrm{mg} / \mathrm{kg}$, tendance observée par Kowalchyk \& Olson 
(1979), et plus rapide entre 40 et 160 $\mathrm{mg} / \mathrm{kg}$. Le $\mathrm{pH}$ du lait ayant été ajusté, les variations du temps de latence jusqu'à 160 $\mathrm{mg} / \mathrm{kg}$ traduiraient bien une accélération de la phase d'agrégation. Au-delà de 160 $\mathrm{mg} / \mathrm{kg}$, ce paramètre n'évolue plus. Des résultats comparables ont été obtenus, notamment par Van Hooydonk (1987) et Lee (1986).

En coagulation mixte, il y a peu d'effet de l'apport de calcium sur le temps de latence. Pour expliquer ce phénomène, il est utile d'examiner la répartition probable du calcium entre les phases micellaire et soluble. La quantité de Ca fixée par les micelles, au $\mathrm{pH}$ du lait $(\mathrm{pH} 6,60)$, a été donnée par Brulé (1981) pour des apports en calcium de 0 à $4 \mathrm{~g} / \mathrm{kg}$. En particulier, dans la gamme des faibles apports en calcium (250 mg/kg de calcium fixé pour $386,4 \mathrm{mg} /$ $\mathrm{kg}$ de calcium ajouté), on détermine simplement un coefficient d'interpolation linéaire $\left(\mathrm{Ca}_{\mathrm{fixé}}=0,647 \times \mathrm{Ca}_{\text {ajoutéé }}\right)$, qui permet d'évaluer la répartition entre les phases micellaire et soluble pour 40,200 et $400 \mathrm{mg} / \mathrm{kg}$ (Tableau II). Connaissant par ailleurs l'effet du $\mathrm{pH}$ sur la solubilisation du calcium du lait en l'absence d'apport de calcium (Brulé, 1981; Rajput et al., 1983;
Van Hooydonk, 1986c), on a estimé la répartition $\mathrm{Ca}$ micellaire/Ca soluble à $\mathrm{pH}$ 6,00 dans la lait (Tableau II). On constate alors que la quantité de $\mathrm{Ca}$ soluble dans un lait à $\mathrm{pH} 6,00$ est supérieure à celle d'un lait additionné de $400 \mathrm{mg} / \mathrm{kg}$ de $\mathrm{Ca}$ à $\mathrm{pH}$ 6,60 : par conséquent, tout ajout de $\mathrm{CaCl}_{2}$ au lait emprésuré à $\mathrm{pH}$ 6,00 augmente cette fraction soluble. Dans le Tableau II, on a estimé enfin les quantités de calcium soluble à $\mathrm{pH} 6,00$ et pour différents niveaux d'apports en calcium, en supposant que les variations de cette fraction dépendaient seulement de la quantité de $\mathrm{CaCl}_{2}$ ajouté et non du $\mathrm{pH}$, hypothèse que les résultats de Van Hooydonk et al. (1986a) confirmeraient. En analysant les résultats obtenus en coagulation enzymatique par rapport au calcium soluble, on constate que lorsque cette fraction est $>450 \mathrm{mg} / \mathrm{kg}$ environ, le temps de latence n'est plus sensible à l'ajout du calcium. Or, les résultats observés en coagulation mixte correspondent précisément à cette situation avant même tout ajout de $\mathrm{CaCl}_{2}$, ce qui expliquerait l'absence d'effet du $\mathrm{CaCl}_{2}$ ajouté sur le temps de latence.

En coagulation enzymatique, jusqu'à 40 $\mathrm{mg} / \mathrm{kg}$, la contrainte maximale augmente

Tableau II. Répartition du calcium entre les phases micellaire et soluble (calculée d'après les résultats de Brulé, 1981, indiqués en caractères gras). $\mathrm{Ca}_{\mathrm{T}}$ : calcium total $(\mathrm{g} / \mathrm{l}) ; \mathrm{Ca}_{\mathrm{m}}$; calcium micellaire $(\mathrm{g} / \mathrm{l})$; $\mathrm{Ca}_{\mathrm{s}}$ : calcium soluble $(\mathrm{g} / \mathrm{l})$.

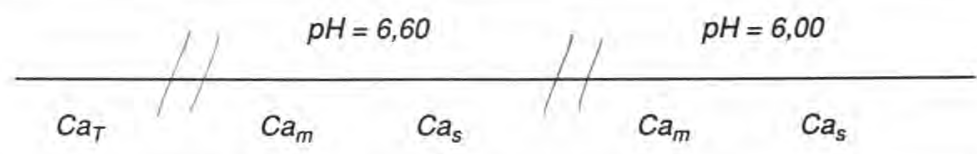

\begin{tabular}{llllll}
\hline Lait & $\mathbf{1 , 2 5}$ & $\mathbf{0 , 8 5 0}$ & 0,400 & 0,696 & $\mathbf{0 , 5 5 4}$ \\
$+0,040 \mathrm{~g}(\mathrm{Ca}) / \mathrm{kg}$ & 1,29 & 0,876 & 0,414 & 0,717 & 0,573 \\
$+0,200 \mathrm{~g}(\mathrm{Ca}) / \mathrm{kg}$ & 1,45 & 0,979 & 0,471 & 0,799 & 0,651 \\
$+0,400 \mathrm{~g}(\mathrm{Ca}) / \mathrm{kg}$ & 1,65 & 1,109 & 0,541 & 0,901 & 0,749 \\
\hline
\end{tabular}


modérément. En revanche, la vitesse maximale de raffermissement diminue sensiblement, confirmant les observations de Kowalchyk \& Olson (1979). Un ajout de calcium limité à $40 \mathrm{mg} / \mathrm{kg}$ se manifesterait essentiellement par une diminution de la vitesse d'organisation du gel.

Entre 40 et $160 \mathrm{mg} / \mathrm{kg}$ de Ca ajouté, la contrainte maximale et la vitesse maximale de raffermissement augmentent, indiquant à la fois un effet positif sur la vitesse d'organisation du réseau ainsi qu'une augmentation de la fermeté. L'ajout de calcium se traduirait par une fixation intramoléculaire, entraînant une diminution de la charge nette des micelles (Dalgleish, 1984) et une diminution de l'hydratation (Baumy \& Brulé, 1986), pour finalement entraîner une formation plus rapide d'un gel plus ferme. On peut en effet penser que le calcium favorise le pontage entre les agrégats (Baumy \& Brulé, 1986), donc l'organisation du gel. Au-delà de $160 \mathrm{mg} /$ $\mathrm{kg}$, la contrainte maximale et la vitesse maximale de raffermissement baissent fortement. Tandis que la phase d'agrégation n'est plus affectée par les ajouts de calcium, l'organisation du réseau est fortement ralentie et conduit à l'obtention d'un gel plus mou. On peut penser que le calcium bloque certains sites réactifs par fixation intermoléculaire (Baumy \& Brulé, 1986), en particulier sur les caséines $\alpha_{s}$ et $\beta$ (Mac Mahon et al., 1984), sites qui deviennent indisponibles pour la réticulation et gênent la coagulation en conduisant à des effets retard (Mac Mahon et al., 1984; Patel \& Reuter, 1986). De plus, l'ajout concomitant des ions chlorure entraîne une augmentation de la force ionique, diminuant les liaisons électrostatiques contribuant à limiter le degré d'organisation du gel.

En coagulation mixte, on remarque, d'une part, qu'une addition de calcium $>300 \mathrm{mg} / \mathrm{kg}$ environ conduit à des teneurs en calcium micellaire comparables à celles qui peuvent être observées sur des laits à $\mathrm{pH} 6,60$, additionnés ou non de calcium (Tableau II).

D'autre part, la vitesse de raffermissement et la contrainte au point $P_{M}$ présentent des valeurs du même ordre de grandeur que les paramètres équivalents en coagulation enzymatique. Ces résultats pourraient suggérer que l'ajout de calcium, en limitant la déminéralisation des micelles due à l'acidification, empêcherait à $\mathrm{pH}$ 6,00 la libération de certains sites actifs pour la réticulation du coagulum. Ce phénomène expliquerait alors la diminution de la fermeté et de la vitesse de raffermissement pour des quantités importantes de calcium ajouté (Fig. 3d). Des résultats analogues ont été observés par Marshall et al. (1982).

L'évolution de l'angle de perte, dans le cas de la coagulation enzymatique, n'est pas sensible à l'ajout de calcium. Ce résultat a aussi été observé dans le domaine linéaire par Bohlin et al. (1984), ainsi que par Zoon et al. (1988). On peut donc raisonnablement penser que ce résultat n'est pas affecté par les conditions de l'essai harmonique, domaine linéaire ou non. En coagulation mixte, l'évolution de l'angle de perte en $P_{M}$ et en $P_{m}$ en fonction de la concentration en calcium indiquerait que la structure du coagulum est légèrement plus élastique à 40 ou $80 \mathrm{mg} / \mathrm{kg}$ de $\mathrm{CaCl}_{2}$ ajouté qu'en l'absence d'addition, mais que cette caractéristique est ensuite peu modifiée par la quantité de calcium ajouté.

L'étude de l'influence de l'addition de calcium suggère que le meilleur compromis entre la fermeté du coagulum, son élasticité et la vitesse de raffermissement est obtenu au voisinage de :

$-160 \mathrm{mg} / \mathrm{kg}$ de $\mathrm{Ca}$ pour un coagulation enzymatique à $\mathrm{pH} 6,60$;

- et de $40 \mathrm{mg} / \mathrm{kg}$ de $\mathrm{Ca}$ en coagulation mixte avec emprésurage à $\mathrm{pH} 6,00$. 
Le raffermissement est un peu moins rapide sans adjonction de calcium, mais le coagulum est moins ferme et moins élastique. Jusqu'à $160 \mathrm{mg} / \mathrm{kg}$, la fermeté et l'élasticité sont peu modifiées mais le ralentissement de la cinétique est sensible. Au-delà de $160 \mathrm{mg} / \mathrm{kg}$, le ralentissement est de plus en plus marqué, la fermeté du coagulum diminue alors que son élasticité varie peu. Jager (1986) a aussi montré les effets négatifs en fromagerie d'une addition trop importante de calcium. II est intéressant de noter que la pratique fromagère courante recommande l'ajout de $40 \mathrm{mg} / \mathrm{kg}$ de $\mathrm{CaCl}_{2}$, exceptionnellement $80 \mathrm{mg} / \mathrm{kg}$, limite fixée par la législation française.

\section{CONCLUSION}

Ces premiers résultats montrent l'intérêt de l'analyse rhéologique, et en particulier avec un viscoélasticimètre du type GVTINRA, pour l'étude des phénomènes liés à la coagulation du lait. La possibilité de caractériser, d'une part, les cinétiques de coagulation à la fois sur le plan de la fermeté et de la viscoélasticité, et d'évaluer d'autre part la vitesse de raffermissement, apporte un ensemble d'informations qui, associées à l'examen des effets des paramètres technologiques, devrait conduire à une meilleure compréhension des mécanismes mis en jeu. Dans le cas de la coagulation mixte, même si en fromagerie le temps de décaillage intervient avant la réorganisation du réseau protéique, ce type d'étude, associé à la connaissance de l'évolution $\mathrm{du} \mathrm{pH}$ et de la répartition du calcium entre les formes micellaire et soluble (ionique ou non), pourrait notamment contribuer à mieux cerner le potentiel d'évolution du grain de caillé lors de l'égouttage.

Enfin, nos résultats confirment la nécessité de raisonner sur la fraction de calcium soluble et non sur la quantité de calcium ajouté pour comprendre les mécanismes de la coagulation.

\section{REMERCIEMENTS}

Nous sommes particulièrement reconnaissants à R. Grappin, directeur de la station de recherches en technologie et analyses laitières (INRA-Poligny) ainsi qu'au Pr G. Brulé (ENSARennes) pour leurs conseils et les discussions critiques lors de la rédaction de cet article.

Nous remercions spécialement $\mathrm{S}$. Aligé et $\mathrm{J}$. Rault (laboratoire de génie de l'hygiène et des procédés alimentaires, INRA-Massy) pour leur assistance technique.

\section{RÉFÉRENCES}

Baumy J.J. \& Brulé G. (1986) Etude comparée de la solubilité et de la viscosité des solutions de caséinate et paracaséinate de sodium en présence de calcium. Lait 66, 65-77

Bohlin L., Hegg P.O. \& Ljusberg-Wahren H.L. (1984) Viscoelastic properties of coagulating milk. J. Dairy Sci. 67, 729-734

Bringe N.A. \& Kinsella J.E. (1986) Influence of calcium chloride on the chymosin-initiated coagulation of casein micelles. J. Dairy Res. 53, 371-379

Brulé G. (1981) Les minéraux du lait. Rev. Lait. Fr. 400, 61-65

Dalgleish D.G. (1984) Measurement of electrophoretic mobilities and zeta-potentials of particles from milk using laser Döppler electrophoresis. J. Dairy Res. 51, 425-438

Dejmek P. (1987) Dynamic rheology of rennet curd. J. Dairy Sci. 70, 1325-1330

Evenhuis N. \& De Vries T.M.R. (1959) The condition of calcium phosphate in milk. Neth. Milk Dairy J. 13, 1-15

Famelart M.H.(1982) Facteurs physicochimiques de la gélification du lait par la pré- 
sure. DEA sci. alimentaires, Paris VII Paris XIENSIA

FIL(1987) Determination of chymosin and bovine pepsin contents. Norme FIL $110 \mathrm{~A}$

Gervais A., Vermeire D., Cerf O. \& Toux J. (1982a) Procédé et appareil de mesure des propriétés rhéologiques de corps semi-solides par cisaillement harmonique de rotation. Demande de brevet français $n^{\circ} 82.17835$, des Etats-Unis $n^{\circ}$ 7130-US-2712, européen (Allemagne, Grande-Bretagne, Pays-Bas, Italie) $n^{\circ}$ 83.402065.3 (déposé par l'INRA)

Gervais A., Vermeire D., Cerf O. \& Toux J. (1982b) Caractère viscoélastique non linéaire du caillé présure. XXIe Congr. Int. Lait. Moscou $1,180-181$

Green M.L. (1977) Review of the progress of dairy science : milk coagulants. J. Dairy Res. 44, 159-188

Heertje I., Visser J. \& Smits P. (1985) Structure formation in acid milk gels. Food Microstruct. 4, 267-277

Jager H. (1986) Das Calcium und sein Einfluss in der Käserei. Dtsch. Milchwirtsch. 37, 496-498

Kobayashi M., Ishikawa S. \& Samejima M. (1982) Application of non linear viscoelastic analysis by the oscillation method to some pharmaceutical ointments in the Japanese pharmacopeia. Chem. Pharm. Bull. 30, 4468-4478

Kowalchyk A.W. \& Oison N.F.(1979) Milk clotting and curd firmness as affected by type of milk clotting enzyme, calcium chloride concentration and season of year. J. Dairy Sci. 62, 1233-1237

Lee K.T.(1986) Contribution à l'étude cinétique de la coagulation du lait par la présure : comparaison de deux méthodes rhéologiques. Thèse de docteur-ingénieur, Paris VII-Paris XI-ENSIA

Lefèvre V. (1988) Etude rhéologique de la formation des gels de caséines obtenus par traitement enzymatique. Thèse phys., Paris VII

Lehembre N. (1986) Contribution à l'étude de la cinétique de coagulation mixte d'un lait reconstitué suivie par une méthode rhéologique : effet du calcium et étude multifactorielle de l'influence de la température, $\mathrm{du} \mathrm{pH}$ et de la dose de présure. ISAA science et technologie alimentaire. INA Paris-Grignon. DAA. ENSA Toulouse.

McMahon D.J., Brown R.J., Richardson G.H. \& Ernstrom C.A. (1984) Effects of calcium, phos- phate and bulk culture media on milk coagulation properties. J. Dairy Sci. 67, 930-938

Marshall R.J., Hatfield D.S. \& Green M.L. (1982) Assessment of two instruments for continuous measurement of the curd-firming of renneted milk. J. Dairy Res. 49, 127-135

Patel R.S. \& Reuter H. (1986) Effect of sodium, calcium and phosphate on properties of rennet coagulated milk. Lebensm.-Wiss. Technol. 19, 288-291

Prokopek D. (1978) Bestimmung der Festigkeit der Gallerte beider Käscherstellung. Dtsch. Milchwirtsch. 29, 534-543

Rajput Y.S., Bhavasasan M.K. \& Ganguli N.C. (1983) Changes in the chemical status of calcium in casein micelles with the $\mathrm{pH}$ of milk. Milchwissenschaft 38, 211-213

Roefs S.P.F.M. (1986) Structure of acid casein gels. A study of gels formed after acidification in the cold. Thesis, Agricultural University, Wageningen, Netherlands

Thomasow J. \& Voss E. (1977) Methods for the determination of the firmness of milk coagulum. Bull. Fed. Int. Lait. 99, 1-9

Van Hooydonk A.C.M. (1987) The renneting of milk. A kinetic study of the enzymic and aggregation reactions. Thesis $\mathrm{Ph}$. D., Université de Wageningen, Pays-Bas

Van Hooydonk A.C.M., Hagedoorn H.G. \& Boerrigter I.J. (1986a) pH-induced physico-chemical changes of casein micelles in milk and their effect on renneting. 1. Effect of acidification on physico-chemical properties. Neth. Milk Dairy J. 40, 281-296

Van Hooydonk A.C.M., Boerrigter I.J. \& Hagedoorn H.G. (1986b) pH-induced physicochemical changes of casein micelles in milk and their effect on renneting. 2. Effect of $\mathrm{pH}$ on renneting of milk. Neth. Milk Dairy J. 40, 297-313

Van Hooydonk A.C.M., Boerrigter I.J. \& Hagedoorn H.G. (1986c) The effect of various cations on the renneting of milk. Neth. Milk Dairy J. 40, 369-390

Walstra P. \& Van Vliet T. (1986) The physical chemistry of curd making. Neth. Milk Dairy J. 40, 241-259

Zoon P., Van Vliet \& Walstra P. (1988) Rheological properties of rennet-induced skim milk gels. 3. The effect of calcium and phosphate. Neth. Milk Dairy J. 42, 295-312 